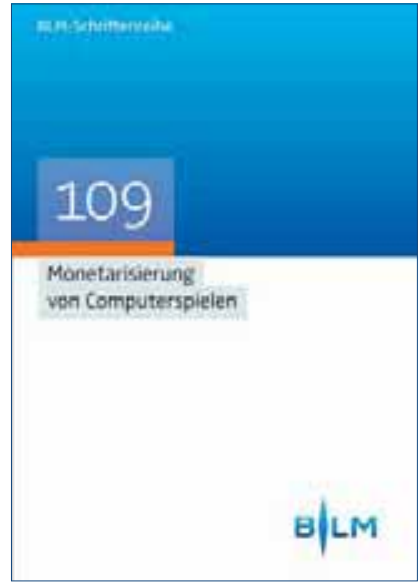

\section{Monetarisierung von Computerspielen}

$$
\text { KOUBEK, Jochen }
$$

Bayerische Landeszentrale für neue Medien (BLM), München Nomos Verlagsgesellschaft, Baden-Baden 2020

\author{
BLM-Schriftenreihe, Band 109 \\ ISBN 978-3-8487-7943-7 \\ 295 Seiten, 39 Euro
}

Der Band "Monetarisierung von Computerspielen" von Jochen Koubek nimmt sich der Ökonomie von Computerspielen an und besteht aus vier Kapiteln: Kapitel 1 „Ökonomie von Computerspielen“, Kapitel 2 „Spielerinnen und Spieler" (welches eine intensive Betrachtung der Spielenden als Kunden der Spielindustrie liefert), Kapitel 3 „Monetarisierungsmodelle“ sowie Kapitel 4 „Fazit" (welches zu dem Ergebnis kommt, dass lediglich verschiedene Formen der In-Game-Transaktionen als substanziell problematisch angesehen werden können, und mögliche Maßnahmen in Richtung Medienkompetenzvermittlung und Jugendmedienschutz formuliert).

Auch wenn, wie Koubek nachvollziehbarer Weise schreibt, die Ökonomie und die Monetarisierungsformen von Computerspielen immer im Kontext der Gestaltung von Spielen sowie der Spielenden zu bewerten sind, sollen in dieser Rezension insbesondere die beiden Kapitel 1 „Ökonomie von Computerspielen" und 3 "Monetarisierungsmodelle" im Fokus stehen. Nicht, weil die anderen Aspekte weniger wichtig wären (im Gegenteil: gerade in der Kombination liegt doch die Herausforderung manipulativer Wirkungen von Computerspielen, insbesondere bei Kindern und Jugendlichen), sondern weil gerade hier ein zusätzlicher Mehrwert (über den Auftrag der BLM hinausgehend) für die Medienökonomie und das Medienmanagement entsteht.
Im Kapitel 1 "Ökonomie von Computerspielen" geht Koubek zunächst knapp auf die äußere Ökonomie (hier geht es um die Einnahmen, welche mit Computerspielen erzielt werden sowie um die Ausgaben im Sinne von Entwicklungskosten) ein, bevor er ausführlich die innere Ökonomie thematisiert. Bei letzterer geht es um den Ressourcenfluss innerhalb eines Spiels (die "Spielökonomie"), welcher unter anderem die Erzeugung, die Verteilung und den Verbrauch virtueller Währungen und Spielgüter (mit Einfluss auf den Spielverlauf) beschreibt. Gerade die Verbindung der inneren Ressourcen-Ökonomie mit der äußeren GeldÖkonomie werden dabei als wesentliches Merkmal moderner Monetarisierungsformen bezeichnet. Hierbei sei insbesondere auf den Abschnitt 1.3.3 verwiesen, welcher die "Hybride Ökonomie" von Computerspielen (auch anhand von Beispielen) vorstellt.

„Eine allgemeine Spielökonomie berücksichtigt neben Geld weitere Ressourcen, darunter Zeit, Aufmerksamkeit, Daten und soziale Kontakte, die für Spiele investiert und bei Kaufentscheidungen handlungsleitend werden" (Seite 21). Entsprechend geht Abschnitt 1.4 auf Aspekte ein, die in direktem Bezug zur Spielökonomie stehen: (1) die sogenannten Loops als Spielhandlungen beziehungsweise Handlungsmöglichkeiten, welche einen Einfluss auf die Ressourcen innerhalb des Spiels haben, (2) das Balancing, welches im Sinne einer Verfeinerung der Spielmechanik letztlich darauf abzielt, den sogenannten Ludic Contract zwischen Spieler*innen und Spielentwickler*innen zu stärken, um die Spielenden an das Spiel „zu binden“, (3) die sogenannten „Dark Patterns", als das von den Spieleentwickler*innen gezielte Herbeiführen negativer Spielerlebnisse mit dem Ziel der Monetarisierung, in dem von den Spielenden Zeit, Geld und Sozialkapital aufgebracht werden muss, um diese negativen Spielerlebnisse zu vermeiden, (4) Glücksspiele, welche viele Merkmale der inneren Ökonomien von Computerspielen gemein haben.

Kapitel 3 "Monetarisierungsmodelle" geht zunächst (Abschnitt 3.1) auf die möglichen Bezahlverfahren ein, welche im Rahmen von Computerspielen möglich sind: Bargeld, Prepaid, Debit- oder Guthabenkarten und Mobile Payment, 
Überweisung, Bankeinzug und Kreditkarte, elektronische Zahlungsmittel wie PayPal, die Bezahlung in Form der Vergütung mit virtuellen Gütern, denen ein realer Gegenwert zugeordnet werden kann sowie Blockchain-Technologien. Es folgen sechs Abschnitte (3.2 bis 3.7), welche in beeindruckender Detailtiefe (auf knapp 100 Seiten) die sechs Monetarisierungsmodelle Subscription (Abonnement), Advertising (Werbung), Affiliate (Partnernetzwerke), Sales (Verkauf), Transaction Fee (Transaktionsgebühren) und Freemium, jeweils mit entsprechenden Unterformen und sowohl allgemein, insbesondere aber bezogen auf Computerspiele, thematisieren. Bei Kapitel 3 handelt es sich somit um eine systematische und vollständige Aufbereitung der möglichen Bezahlverfahren und Monetarisierungsmodelle in der Spieleindustrie.

Genau dabei entsteht der oben erwähnte zusätzliche Mehrwert für die Medienökonomie und das Medienmanagement - welcher nämlich in genau dieser systematischen und vollständigen Aufbereitung der möglichen Bezahlverfahren und Monetarisierungsmodelle liegt - eben nicht nur für die Spieleindustrie, sondern generell für (insbesondere digitale) Medien(inhalte). Insofern eignet sich der Band „Monetarisierung von Computerspielen", welcher einen wertvollen Beitrag zum Diskurs Geschäftsmodelle im Gaming-Bereich liefert, eben nicht nur für Politik, Entscheider*innen und Gestalter*innen sowie Wissenschaftler*innen mit Interessengebiet "Computerspiele“, sondern für alle, die sich mit Monetarisierungsmodellen und Erlösmodellen in der Medienwirtschaft beschäftigen.

Es ist anzumerken, dass der Band stets mit konkreten Beispielen aus der Spielebranche arbeitet und dass im Rahmen der Studie mit zehn Experten aus der Branche sowie aus Forschung und Lehre zu den Feldern Gamedesign, Vermarktung und Monetarisierung von Computerspielen sowie Konsumentenverhalten gesprochen wurde. Auszüge der Interviews sind den entsprechenden Stellen des Buches zugeordnet und dort nachzulesen.

Prof. Dr. Uwe Eisenbeis Hochschule der Medien Stuttgart 